\title{
Aceros rápidos reforzados con carburos mediante molino de alta energía
}

\author{
J.M. RUIZ-ROMÁN, L.E.G. CAMBRONERO, J.C. SUÁREZ1 , F. CORPAS² Y J.M. RUIZ-PRIETO \\ Universidad Politécnica de Madrid. E.T.S.I. Minas, Rios Rosas 21, 28003 Madrid. \\ $(*)$ Universidad Politécnica de Madrid. E.T.S.I. Navales, Ciudad Universitaria 28040, Madrid \\ $(* *)$ Universidad de Jaén. E.U.P. Linares. Alfonso X el Sabio 28, 23700 Linares
}

\begin{abstract}
En este trabajo se estudia la eficacia del empleo de la aleación mecánica, en el proceso de fabricación de materiales compuestos de matriz metálica (base acero rápido M2) mediante técnicas pulvimetalúrgicas. Del análisis de los materiales obtenidos, acero rápido $\mathrm{M} 2$ reforzado con $\mathrm{WC}$, se deduce que la aleación mecánica resulta ser un método eficaz para la fabricación de estos compuestos, ya que se alcanzan unas excelentes propiedades mecánicas, que superan ampliamente a las que presentan los mismos aceros reforzados con carburos mediante procesos pulvimetalúrgicos convencionales.

\section{Palabras clave: Acero rápido, Aleación mecánica, Materiales compuestos de matriz metálica}

\section{High speed steels reinforced with carbides by mechanical alloy}

In this research work we have studied the efficiency of using an advanced techniqhe, as mechanical alloying, on the metal matrix composites fabrication process by powder metallurgy. From the analysis of several composites, based on M2 high speed steel reinforced with WC, we inferred that mechanical alloying is an effectve method to manufacture these materials, and they present higher mechanical properties than high alloy steels used in structural applications.
\end{abstract}

Key words: High speed steel, Mechanical Alloy, Metal matrix composite

\section{INTRODUCCIÓN}

Los aceros rápidos sinterizados presentan unos inconvenientes que la Metalurgia de Polvos intenta resolver. En primer lugar las altas temperaturas de sinterización requeridas para obtener dichos materiales y en segundo lugar la fuerte competencia que se les presenta a estos aceros por otros materiales entre los que hay que resaltar a los Cermets. En esta segunda vía, lo que se pretende con este trabajo de investigación es reforzar estos aceros con porcentajes diferentes de carburos (1) con el fin de elevar sus propiedades. Para ello se ha utilizado la aleación mecánica (2) con el objeto de mejorar la cohesión entre la matriz y el refuerzo que constituyen los materiales compuestos fabricados en este trabajo $(3,4)$. La experiencia del equipo investigador (5) en el refuerzo de materiales sinterizados condujo a la necesidad de emplear la aleación mecánica como único método eficaz para la mezcla y unión de los refuerzos (carburo deWolframio) con la matriz de acero rápido, en este caso el acero rápido M2.

\section{PROCEDIMIENTO EXPERIMENTAL}

Para la obtención de los materiales base acero rápido M2 reforzados con diferentes proporciones de WC $(5 \%, 10 \%, 15 \%)$ se siguió el proceso que se detalla a continuación:

\subsection{Materias primas}

Para la preparación del material se han empleado como materias primas polvo de acero rápido $\mathrm{M} 2$ y polvo de carburo de wolframio de las siguientes características:

Polvo de acero rápido M2 de la casa COLDSTREAM, fabri-
TABLA I. COMPOSICIÓN QUíMICA DEL POLVO DE ACERO RÁPIDO M2 DE LA CASA COLDSTREAM.

\begin{tabular}{|c|c|c|c|c|c|c|c|}
\hline \%C & \%Mo & \%W & \%Fe & \%Mn & \% Cr & \%Si & \%V \\
\hline 0,88 & 4,96 & 6,44 & Base & 0,19 & 4,09 & 0,21 & 1,92 \\
\hline
\end{tabular}

cado mediante atomización con gas y molienda. Su composición química se recoge en la tabla I, y sus propiedades físicas son las siguientes: densidad aparente $2,24 \mathrm{~g} / \mathrm{cm}^{3}$; velocidad de flujo 38,9 s/50g y tamaño de partícula inferior a $150 \mu \mathrm{m}$.

Polvo de WC de la casa GOODFELLOW, con las siguientes características: densidad aparente $15,63 \mathrm{~g} / \mathrm{cm}^{3}$, pureza 99,5\%; tamaño medio de partícula 150 y tamaño mínimo de 45 .

\subsection{Aleación Mecánica}

La composición de la mezcla inicial, o mezcla madre, obtenida mediante aleado mecánico fue de un $75 \%$ en masa de polvo de acero rápido M2 y de un $25 \%$ de polvo de WC. Para conseguirla se empleó un molino de alta energía (attritor), teniendo el proceso las siguientes características:

-Cuerpos moledores: bolas de acero inoxidable

- Carga de cuerpos moledores: 10 x carga de polvos

- Tiempo de molienda 1 hora

—Velocidad del molino: $500 \mathrm{rpm}$

La granulometría de la mezcla obtenida mediante aleación mecánica se recoge en la tabla II.

TABla II. GRANUlOMETRÍA DE LA MEZCla OBTENIDA POR ALEACIÓN MECÁNICA.

\begin{tabular}{|c|c|c|c|c|c|c|}
\hline Tamaño de partícula $(\mu \mathrm{m})$ & $>180$ & 80 & 63 & 53 & 45 & $<45$ \\
\hline Composición de la mezcla $(\%)$ & 6,2 & 33,4 & 10,6 & 0,6 & 7,1 & 42,1 \\
\hline
\end{tabular}




\subsection{Preparación de las muestras}

Una vez obtenida la mezcla inicial en el molino de alta energía, se procede a su mezclado manual con polvos de acero rápido para la obtención de las proporciones finales seleccionadas, es decir, 5\%, 10\% y 15\% de carburo de Wolframio adicionado como refuerzo. Con este sistema mixto de mezcla, proporcionamos al conjunto la capacidad de generar las uniones mecánicas que dan cohesión al compacto en verde, ya que el polvo obtenido directamente del attritor presenta un grado de acritud tan alto que para poder compactarlo en matriz por si mismo, sería necesario someterlo a un recocido que aumentase su plasticidad.

Una vez obtenidas las mezclas definitivas, la consolidación de los polvos se realizó en una prensa de 150 toneladas mediante compactación uniaxial en matriz flotante, con lubricación en las paredes de la misma y auxiliada por el empleo de un punzón superior. La compactación se realizó a $700 \mathrm{MPa}$, y como agente lubricante se empleó estearato de zinc.

A continuación se sinterizaron los compactos en verde del siguiente modo:

-Calentamiento: $5^{\circ} \mathrm{C} / \mathrm{min}$ hasta $1240{ }^{\circ} \mathrm{C}$

-Mantenimiento: $30 \mathrm{~min}$ a $1240^{\circ} \mathrm{C}$

-Enfriamiento: $10^{\circ} \mathrm{C} / \mathrm{min}$ hasta $\mathrm{T}_{\text {ambiente }}$

-Tipo de atmósfera: Vacío

Por último fueron evaluadas propiedades físicas (densidad y variación dimensional) y de de las propiedades mecánicas fundamentales en un acero de herramientas: la dureza y la resistencia a flexión de los materiales sinterizados resultantes.

Para evaluar la dureza, y debido a la elevada dureza de este tipo de materiales, se empleo la escala Rockwell C, es decir, penetrador de cono de diamante y carga aplicada de $150 \mathrm{~kg}$. En cuanto a la evaluación de la resistencia a flexión en los materiales obtenidos, se realizó según la Norma ASTM B528 para un ensayo de flexión en tres puntos.

En la figura 1 queda recogido el esquema del proceso de fabricación de los materiales obtenidos.

\section{RESULTADOS Y DISCUSIÓN.}

En la tabla III se recogen los resultados de las propiedades físicas evaluadas en los materiales resultantes. Puede apreciarse que la variación dimensional va siendo mayor en valor absoluto aunque negativa, ya que el material contrae al sinterizar tanto más cuanto mayor es el contenido de carburo de wolframio adicionado. Este hecho puede explicarse conjuntamente con el aumento de densidad que se produce en estos materiales a medida que se incrementa su contenido en carburo de wolframio, y que no es simplemente debido al hecho de la mayor densidad del WC, sino que la posible presencia de carbono libre junto con el carburo, debido a posibles contaminaciones del polvo de WC, disminuiría la temperatura de sinterización de la matriz provocando una disminución de porosidad al sinterizar a la misma temperatura.

En las figuras 2 y 3 se pueden observar los resultados obtenidos para las propiedades mecánicas

Como cabría esperar al reforzar eficazmente estos aceros, la dureza aumenta con el contenido de carburo de wolframio por dos motivos:

Una mayor cantidad de carburos presentes en el acero rápido base

La unión entre el refuerzo y la matriz es fuerte y ejerce un efecto positivo en el comportamiento mecánico del material.
TABla III. DENSIDAD Y VARIACIÓN DIMENSIONAL DE lOS MATERIALES COMPUESTOS ACERO RÁPIDO M2 - CARBURO DE WOLFRAMIO SINTERIZADOS A $1240^{\circ} \mathrm{C}$ EN VACÍO.

\begin{tabular}{|c|c|c|c|}
\hline MATERIAL & Var. $\mathbf{d i m} . \mathbf{( \% )}$ & $\rho_{\text {verde }}\left(\mathbf{g} / \mathbf{c m}^{\mathbf{3}}\right)$ & $\rho_{\text {sinterizada }}\left(\mathbf{g} / \mathbf{c m}^{\mathbf{3}}\right)$ \\
\hline M2 $-5 \%$ WC & $-2,91$ & 6,48 & 7,08 \\
\hline M2-10\% WC & $-6,64$ & 6,60 & 8,07 \\
\hline M2-15\% WC & $-6,75$ & 6,68 & 8,12 \\
\hline
\end{tabular}

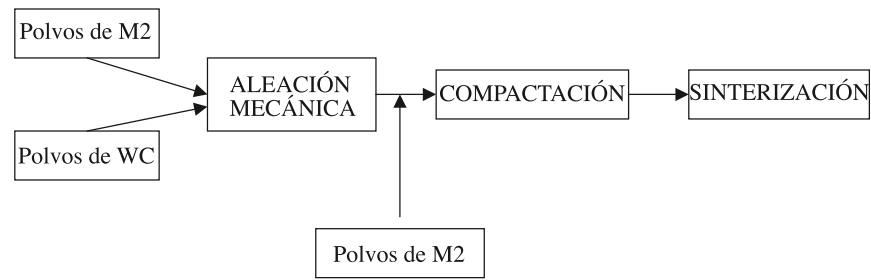

Figura 1. Esquema seguido para la fabricación de los aceros rápidos reforzados con carburo de wolframio.

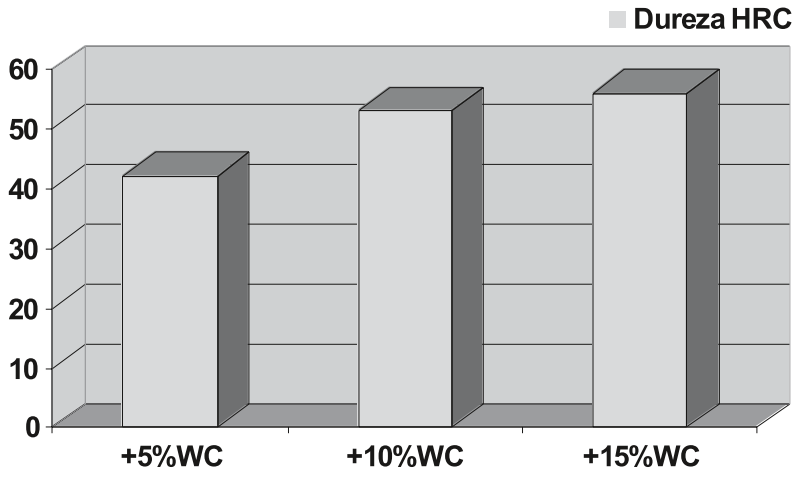

Figura 2. Dureza de los materiales compuestos M2-WC en función del contenido en carburo de wolframio adicionado.

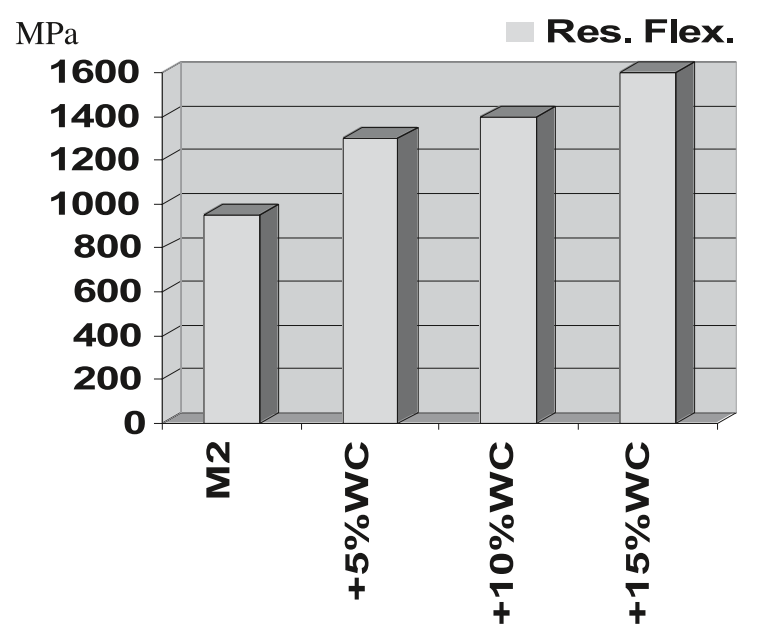

Figura 3. Resistencia a flexión de los materiales compuestos M2-WC en función del contenido en carburo de wolframio adicionado. 
Del mismo modo, y por las mismas razones anteriores se puede observar en la figura 3 como la resistencia a flexión aumenta con el contenido de WC adicionado como refuerzo al acero rápido M2. A estas dos razones anteriores debe añadirse que los carburos resultantes del proceso de aleación mecánica son de pequeño tamaño y están muy dispersos lo que contribuye también al aumento de las propiedades mecánicas.

El estudio microestructural realizado mediante microscopía electrónica de barrido confirma lo descrito en el párrafo anterior. En la figura 4 se puede apreciar la microestructura de un acero rápido $\mathrm{M} 2$ reforzado con un 10\% de Carburo de Wolframio mediante aleación mecánica. Se observa como el proceso de aleación mecánica "incrusta" numerosos carburos de wolframio de tamaño incluso inferior a 1 por toda la matriz generando ese proceso de refuerzo eficaz anteriormente descrito.

En la figura 5 tenemos un detalle de la microestructura del mismo acero, donde se puede apreciar la típica estructura lajosa de los materiales sinterizados a partir de polvo aleado mecánicamente y partículas de carburos de wolframio de diferentes tamaños distribuidos adecuadamente en la matriz.

Es de suponer que las propiedades mecánicas evaluadas de estos materiales se puedan incrementar aún más aplicándoles el tratamiento térmico correspondiente de temple y revenido que debe estudiarse, ya que no debe olvidarse que estas propiedades indicadas en la figura anterior se consiguen con los materiales en su estado previsiblemente mas blando, en estado sinterizado.

\section{CONCLUSIONES}

La mezcla de polvo obtenido por aleación mecánica junto con el polvo sin alear se ha mostrado como un procedimiento eficaz, a la vez que económico, para conseguir compactar materiales sin necesidad de recocidos que eliminen la acritud conferida al polvo aleado mecánicamente.

El material compuesto, acero rápido M2 - carburo de wolframio obtenido mediante aleación mecánica ha permitido obtener un acero de herramientas con unos valores de dureza y resistencia a flexión un 50\% superiores que las que se obtienen con el acero rápido M2 obtenido por técnicas pulvimetalúrgicas convencionales.

Con este trabajo, por tanto, hemos conseguido obtener un material compuesto de matriz metálica con unas propiedades muy superiores a las alcanzadas con los mismos materiales fabricados por procesos convencionales de metalurgia de polvos.

No obstante, los resultados obtenidos previsiblemente se podrían ver mejorados con la adición de $\mathrm{Cu}-\mathrm{P}$ o Fe-P que permitan la formación de fases líquidas con las consiguientes ventajas en el proceso de sinterización.

\section{BIBLIOGRAFÍA}

1. F. Thümmler \& C. Gustfeld. Proc. of the International Conference on Powder Metallurgy, EPMA, Londres, (UK), vol. 2. 25-27, (1990).

2. R.W Gardnier et al. Advanced Performanced Materials, 3 (3/4), 343-359. 1996.

3. F.H. Froes et al. Powder Metallurgy, 39 (1), 63-69, (1996).

4. H. Rosskamp et al. Powder Metallurgy, 39 (1), 37-43, (1996).

5. J.M. Ruiz-Román. Proc. del Congreso Internacional de Tecnología Metalúrgica e de Materiais. Associacao Brasileira de Materiais, Sao Paulo (Brasil). 191-202. (1994).

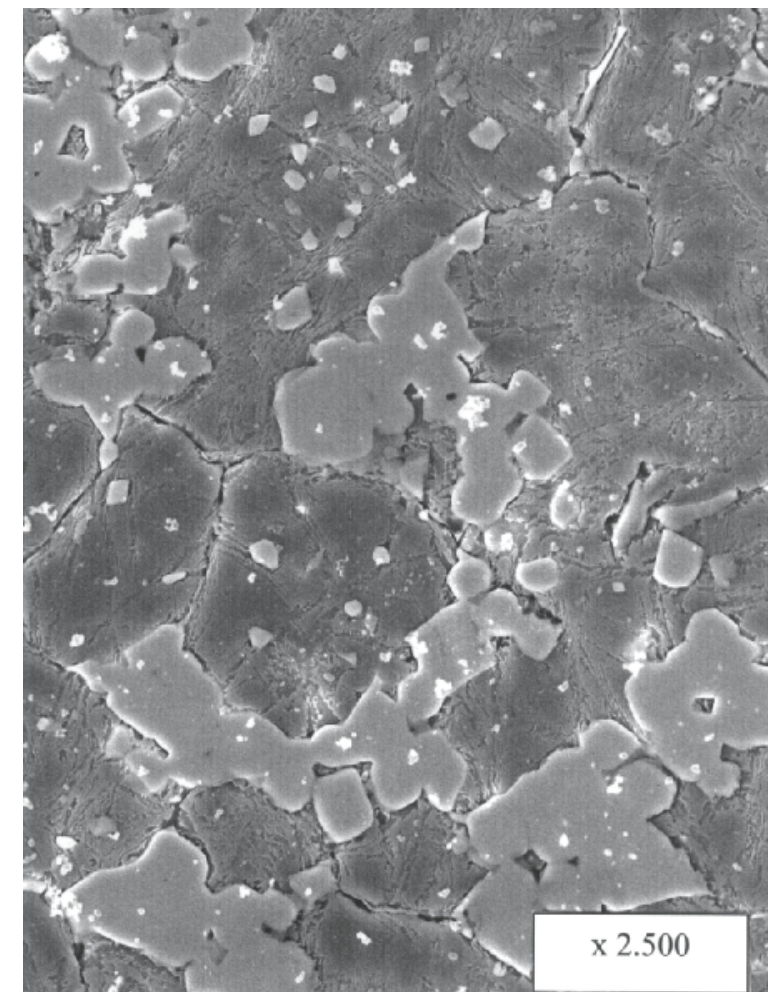

Figura 4. Fotomicrografía de un acero rápido M2 reforzado mediante aleación mecánica con un $10 \%$ de WC. Sinterizada a $1240{ }^{\circ} \mathrm{C}$ en vacío. Atacada con nital.

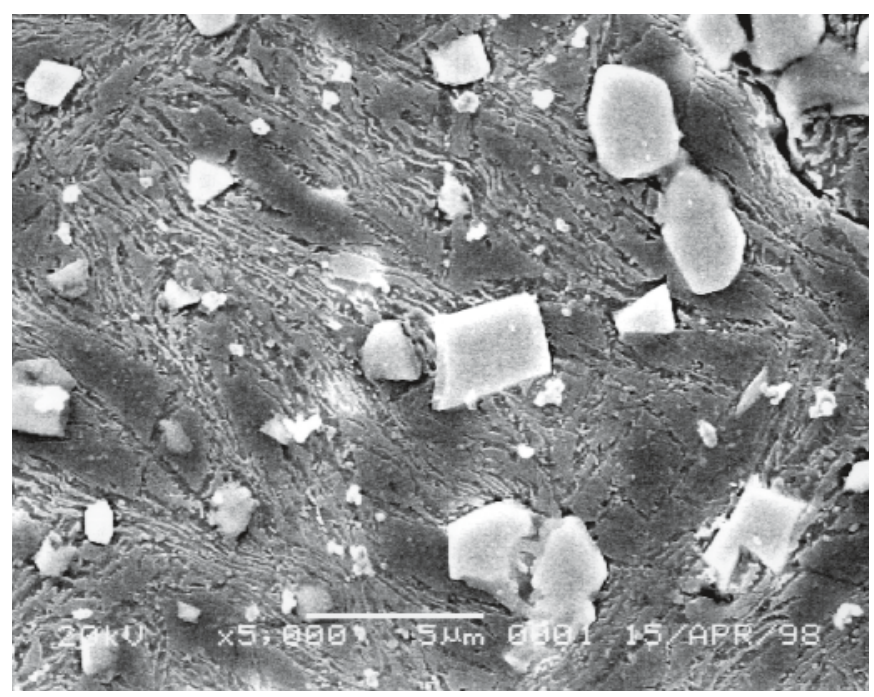

Figura 5. Fotomicrografía de un acero rápido M2 reforzado mediante aleación mecánica con un $10 \%$ de WC. Sinterizada a $1240{ }^{\circ} \mathrm{C}$ en vacío. Atacada con nital. 